\title{
Hepatoid carcinoma of the pancreas mimicking neuroendocrine tumor
}

\section{Kentaro Fukushima, Akira Kobayashi}

First Department of Surgery, Shinshu University School of Medicine, Matsumoto, Japan

Correspondence to: Akira Kobayashi, MD, PhD. First Department of Surgery, Shinshu University School of Medicine, 3-1-1 Asahi, Matsumoto, Nagano 390-8621, Japan. Email: kbys@shinshu-u.ac.jp.

Submitted Jul 09, 2018. Accepted for publication Sep 25, 2018.

doi: $10.21037 /$ hbsn.2018.10.11

View this article at: http://dx.doi.org/10.21037/hbsn.2018.10.11

A 44-year-old man was admitted to our hospital for further investigation of a tumor in the pancreatic head. He had no symptoms or chronic liver diseases. Pancreatic hormones, CEA, and CA19-9 were normal. Serum alpha-fetoprotein and des-gamma-carboxy prothrombin were not assessed.

A contrast-enhanced abdominal CT scan revealed a well-circumscribed, comparatively hypervascular mass during the arterial phase of imaging (panel A), suggesting a neuroendocrine tumor of the pancreas.

The patient underwent tumor enucleation. A macroscopic examination revealed a $19 \mathrm{~mm} \times 18 \mathrm{~mm}$ solid tumor with a fibrous capsule and a nodule-in-nodule appearance (panel B). A H\&E staining revealed the tumor cells contained abundant eosinophilic cytoplasm and exhibited a trabecular pattern with bile production (arrow heads) (panel C, 50x). Immunohistological examinations revealed the tumor cells were positive for Hep Par 1 and glypican 3 (panel D, 50x) and
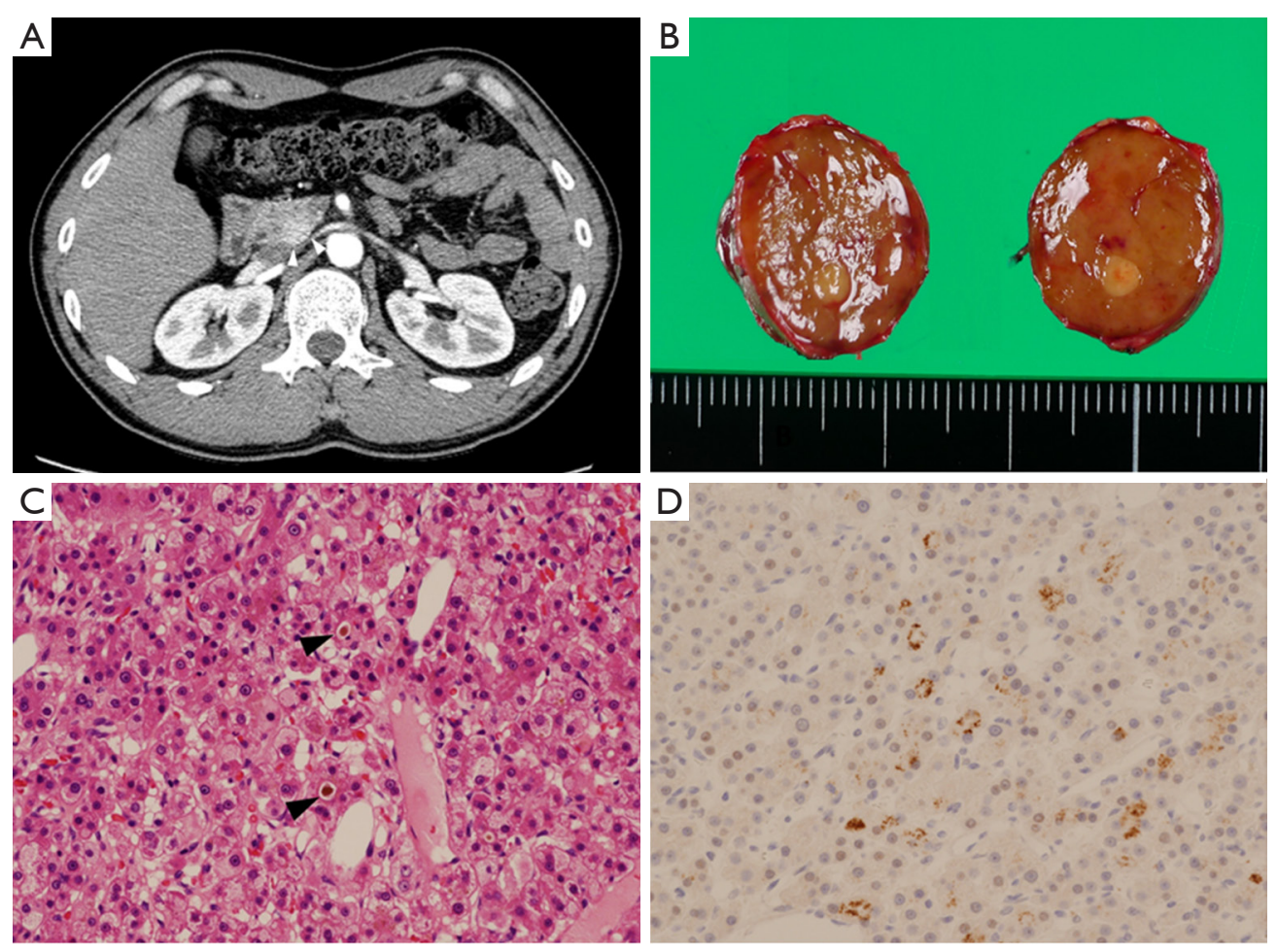
negative for synaptophysin and chromogranin A. We diagnosed the tumor as a hepatoid carcinoma of the pancreas.

\section{Acknowledgements}

The authors thank Dr. Takeshi Uehara, a pathologist at the Department of Laboratory Medicine, Shinshu University Hospital, for his help with the pathological interpretation and final diagnosis.

\section{Footnote}

Conflicts of Interest: The authors have no conflicts of interest to declare.

Informed Consent: Written informed consent was obtained from the patient for publication of this manuscript and any accompanying images.

Cite this article as: Fukushima K, Kobayashi A. Hepatoid carcinoma of the pancreas mimicking neuroendocrine tumor. HepatoBiliary Surg Nutr 2018;7(6):501-502. doi: 10.21037/ hbsn.2018.10.11 